\title{
ATP citrate lyase knockdown impacts cancer stem cells in vitro
}

\author{
J-i Hanai ${ }^{1,2}, N^{2}$ Doro $^{1,4}$, P Seth ${ }^{1}$ and VP Sukhatme $e^{\star, 1,2,3}$
}

ATP citrate lyase (ACL) knockdown (KD) causes tumor suppression and induces differentiation. We have previously reported that ACL KD reverses epithelial-mesenchymal transition (EMT) in lung cancer cells. Because EMT is often associated with processes that induce stemness, we hypothesized that ACL KD impacts cancer stem cells. By assessing tumorsphere formation and expression of stem cell markers, we showed this to be the case in A549 cells, which harbor a Ras mutation, and in two other non-small-cell lung cancer cell lines, H1975 and H1650, driven by activating EGFR mutations. Inducible ACL KD had the same effect as stable ACL KD. Similar effects were noted in another well-characterized Ras-induced mammary model system (HMLER). Moreover, treatment with hydroxycitrate phenocopied the effects of ACL KD, suggesting that the enzymatic activity of ACL was critical. Indeed, acetate treatment reversed the ACL KD phenotype. Having previously established that ACL KD impacts signaling through the phosphatidylinositol 3-kinase (PI3K) pathway, not the Ras-mitogen-activated protein kinase (MAPK) pathway, and that EMT can be reversed by PI3K inhibitors, we were surprised to find that stemness in these systems was maintained through Ras-MAPK signaling, and not via PI3K signaling. Snail is a downstream transcription factor impacted by Ras-MAPK signaling and known to promote EMT and stemness. We found that snail expression was reduced by ACL KD. In tumorigenic HMLER cells, ACL overexpression increased snail expression and stemness, both of which were reduced by ACL KD. Furthermore, ACL could not initiate either tumorigenesis or stemness by itself. ACL and snail proteins interacted and ACL expression regulated the transcriptional activity of snail. Finally, ACL KD counteracted stem cell characteristics induced in diverse cell systems driven by activation of pathways outside of Ras-MAPK signaling. Our findings unveil a novel aspect of ACL function, namely its impact on cancer stemness in a broad range of genetically diverse cell types.

Cell Death and Disease (2013) 4, e696; doi:10.1038/cddis.2013.215; published online 27 June 2013

Subject Category: Cancer Metabolism

Stem-like cells have been identified in a number of human solid tumor types. ${ }^{1}$ They may have a crucial role in tumorigenesis and tumor progression, including tumor metastasis, drug resistance and recurrence. ${ }^{2,3}$ Importantly, stem-like cells, often termed cancer stem cells (CSCs) or tumor-initiating cells, are associated with an aggressive disease process and poor prognosis, ${ }^{4}$ indicating that an understanding of their biology is pertinent to developing effective therapies. ${ }^{5}$

CSCs utilize many of the same signaling pathways that are found in normal stem cells, including Hedgehog, Notch and Wnt signaling. ${ }^{6}$ These embryonic signaling pathways are all known to induce epithelial-mesenchymal transition (EMT) along with factors, such as TGF- $\beta$ and various cytokines. ${ }^{7-10}$ EMT was originally defined as a process of cellular reorganization essential for embryonic development, resulting in the loss of cell-to-cell adhesion, and gain of invasive and migratory mesenchymal properties. ${ }^{11}$ The EMT process is induced not only by embryonic signalings, but also through tumorigenic signaling pathways, such as Ras-mitogenactivated protein kinase (MAPK), phosphatidylinositol 3-kinase (PI3K)-AKT and TGF- $\beta$. Emerging evidence suggests that there is a significant linkage between the EMT process and generation of CSCs. For example, transcriptional factors, such as snail, slug and Twist, have a role in both processes. Their expression also correlates with poor prognosis in cancer patients. ${ }^{12}$

ATP citrate lyase (ACL) connects glucose metabolism to lipid synthesis. It catalyzes the conversion of cytosolic citrate to acetyl-CoA and oxaloacetate, ${ }^{13}$ so as to generate cytosolic acetyl CoA from mitochondrially derived citrate. Cytosolic acetyl-CoA is the requisite building block for endogenous lipid

\footnotetext{
${ }^{1}$ Division of Interdisciplinary Medicine and Biotechnology, Department of Medicine, Beth Israel Deaconess Medical Center and Harvard Medical School, Boston, Massachusetts 02215, USA; ${ }^{2}$ Division of Nephrology, Department of Medicine, Beth Israel Deaconess Medical Center and Harvard Medical School, Boston, Massachusetts 02215, USA and ${ }^{3}$ Division of Hematology-Oncology, Department of Medicine, Beth Israel Deaconess Medical Center and Harvard Medical School, Boston, Massachusetts 02215, USA

${ }^{*}$ Corresponding author: VP Sukhatme, Department of Medicine, Beth Israel Deaconess Medical Center and Harvard Medical School, 330 Brookline Avenue, GZ602, Boston, MA 02215, USA. Tel: + 617667 9050; Fax: + 617667 7843; E-mail: vsukhatm @bidmc.harvard.edu

${ }^{4}$ Current address: College of Osteopathic Medicine, University of New England, Biddeford, 11 Hills Beach Road, ME 04005

Keywords: ATP-citrate lyase; epithelial-mesenchymal transition (EMT); cancer stem cells (CSCs); snail

Abbreviations: ACL, ATP citrate lyase; EMT, epithelial-mesenchymal transition; PI3K, phosphatidylinositol 3-kinase; MAPK, mitogen-activated protein kinase; NSCLC, non-small-cell lung cancer; KD, knockdown; CSC, cancer stem cell; DMEM, Dulbecco's modified Eagle's medium; IP, immunoprecipitation; WB, western blot; FACS, fluorescence-activated cell sorting; PBS, phosphate-buffered saline; MEGM, mammary epithelial growth medium

Received 30.12.12; revised 01.5.13; accepted 14.5.13; Edited by Y Shi
} 
synthesis as well as for acetylation reactions that modify proteins. Growth factors (such as EGF, insulin and PDGF) lead to activation of the PI3K/AKT pathway and this in turn leads to increased enzymatic activity of $A C L$ via AKTmediated ACL phosphorylation, suggesting ACL's role in cell growth and differentiation. A definitive role for $A C L$ in tumorigenesis has emerged from the Thompson group, ${ }^{14,15}$ which reported that decreasing the expression of ACL by shRNA or its activity by a small-molecule inhibitor suppressed tumor growth (leading to a cytostatic effect) and promoted differentiation in numerous glycolytic tumors. ${ }^{14,15}$ We have previously extended these findings and reported that ACL knockdown (KD) in non-small-cell lung cancer (NSCLCs) cells reverses EMT in vitro and in vivo, and suppresses tumor growth, through intercepting PI3K/AKT signaling, but without effect on Ras-MAPK signaling. ${ }^{13}$ Here, we show that ACL KD suppresses cancer stemness in numerous cell types, and does this at least partially through affecting snail expression and function.

\section{Results}

ACL KD reduces stem cell population in NSCLC cells. The EMT is often accompanied by the induction of stemness. ${ }^{16}$ As, we previously reported that ACL KD reverses EMT in NSCLC, ${ }^{13}$ we hypothesized that $A C L$ KD might impact CSCs.

Following published studies, we used the aldefluor ${ }^{17-19}$ assay, and assessed CD133 (general stem cell markers), 20,21 the verapamil (an $A B C$ transporter inhibitor) sensitive side population $^{21-24}$ and CD44 expression (Supplementary Figure 1) to detect lung CSCs, using control and ACL KD cells generated for the human NSCLC cell lines A549, H1975, and $\mathrm{H} 1650 .^{13} \mathrm{~A} 549$ cells bear a K-ras mutation, whereas the last two cell types bear EGFR mutations. H1975 cells harbor two mutations in the EGFR (L858R/T790M) and are gefitinibinsensitive, whereas $\mathrm{H} 1650$ cells carry an activating EGFR mutation caused by a deletion in exon19 (delE746-A750) and are gefitinib-sensitive. Fluorescence-activated cell sorting (FACS) analyses were performed to identify aldefluor-positive or CD133-positive cells (Figure 1a). Similarly, a side population analysis was performed. All three methods consistently showed that the percentage of positive cells was downregulated by $A C L K D$ in each cell line, even though the quantification differed (Figure 1a). To further confirm the effects of ACL KD on lung CSCs, we used the tumorsphere assay. Stable ACL KD showed clear diminution of tumorsphere formation (Figure $1 \mathrm{~b}$ ) in the tumor cell lines. BEAS-2B cells served as negative controls. Inducible ACL KD in A549 cells also showed significant decrement in tumorsphere formation (Figure 1c) and confirmed data from the ACL stable KD cells. Collectively, these data show that ACL KD can impact stem cells in various lung cancer cells driven by either ras activation or by EGFR-activating mutations.

ACL KD affects stem cell populations in a breast cancer cell line. As there exist no definitive markers or absolute isolation methods for stem cells in lung cancer, we chose the well-characterized human mammary breast cancer cell lines
(HMLE and HMLER) $^{25}$ for further experimentation. In this system, CSC markers are well established. HMLE cells are non-transformed mammary epithelial cells and are composed of a single population detected as CD44 negative and CD24 positive (Figure 2a). Constitutively active H-Ras (H-rasV12) transforms HMLE into Ras-transformed cells (HMLER) and induces a new population (stem cell population), detected as CD44 positive and CD24 negative. Rastransformed HMLE (HMLER) cells, therefore, are composed of two populations, a newly induced stem cell population (S) and a non-stem cell population (NS), which can be separately isolated by FACS (Figure 2a). The stem cell population has spindle-shaped cell morphology and expresses mesenchymal markers (vimentin) and forms mammospheres, in contrast to the non-stem cell population (cobble-stone like cells, which express epithelial markers, and exhibit minimal mammosphere formation; Figure 2a). Actually, the stem cell population in HMLER cells (S cells) expresses a higher amount of ACL protein compared with the non-stem cell population (NS cells; Supplementary Figure 2). Using HMLER cells, inducible ACL KD cells (R-ACL KD) and inducible ACL overexpression cells (R-eACL) were generated and processed for FACS analysis. We found downregulation of the stem population in $A C L K D$ cells and upregulation by $A C L$ overexpression (Figure $2 b$ and $c$ ), suggesting that ACL expression levels 'regulate' stem cells. The stem cell population isolated from HMLER cells (S cells) was treated with hydroxycitrate, an enzymatic inhibitor of ACL, and analyzed by flow cytometry. Hydroxycitrate increased the non-stem cell population (Figure 2d) in a dose-dependent manner, suggesting that blocking the enzymatic activity of $A C L$ reduced the stemness at least partially.

MAPK, but not PI3K, signaling is required for maintenance of ras-induced stemness in HMLER cells. In order to clarify which downstream signals mediated by $\mathrm{H}$-Ras generate stem cells in HMLE cells, we treated HMLER cells with a PI3K inhibitor or one or two MEK inhibitors for 3 days. We had anticipated that the PI3K inhibitor would have the maximal impact, as we had previously shown that ACL KD did not affect Ras-MAPK signaling, but inhibited the PI3K pathway and that PI3K inhibitors reversed EMT. ${ }^{26,27}$ As expected, the inhibitors (PI3K inhibitor LY294002 and MEK inhibitor U0126) were effective in downregulating the phosphorylation state of $\mathrm{pAKT}$ and $\mathrm{pERK}$, respectively, in HMLER cells. However, to our surprise, only the MEK inhibitors (U0126 and PD035901) abolished the stem cell population (Figure 3a), in contrast to the impact that both PI3K and MEK inhibitors had on reversing EMT. In these cell lines, cell viability was affected by LY294002 but only at higher concentrations (Supplementary Figure 3). These data suggest that there might be additional ways by which ACL KD affects cancer stemness. We hypothesized that these 'additional point(s)' would be in the MAPK signaling pathway, downstream of MEK, as a MEK inhibitor impacted stemness in this model.

As transcriptional factors, such as snail, slug and twist, promote EMT and stemness, we assessed the expression of these factors in the HMLER cells treated with MEK and PI3K 
a

CD133 (PE)
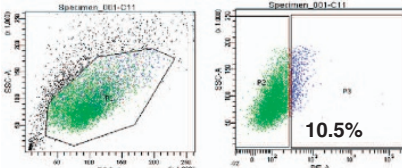

A549 control

Aldefluor
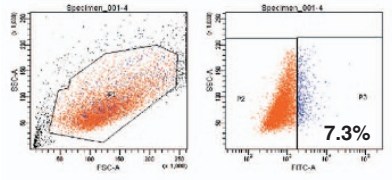

H1975 control

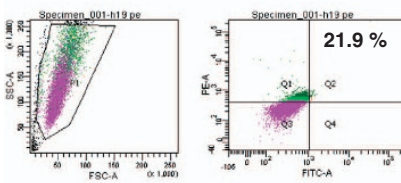

H1975 control

Hoechst 33342 (side population)
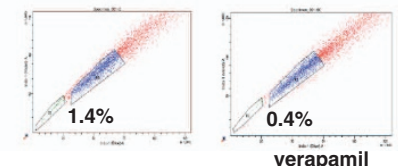

H1650 control

b

b
A549

control

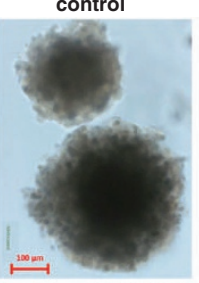

ACL KD
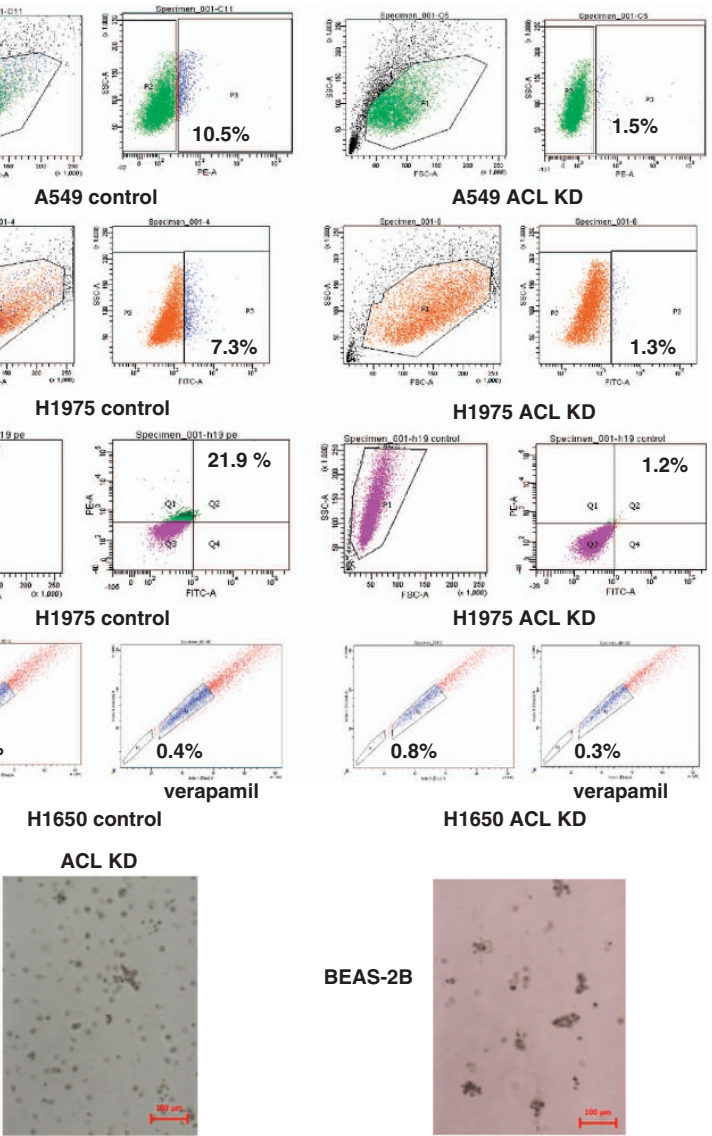

A549 ACL KD

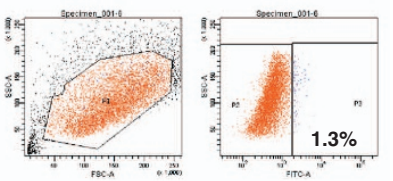

H1975 ACL KD

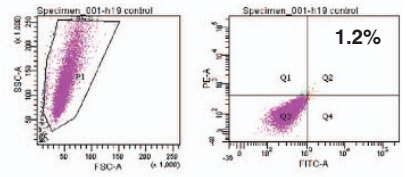

H1975 ACL KD

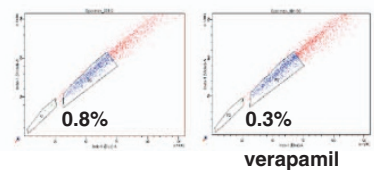

H1650 ACL KD

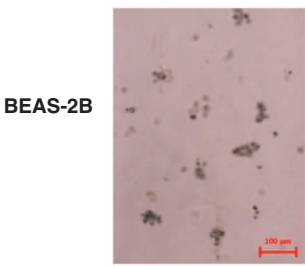

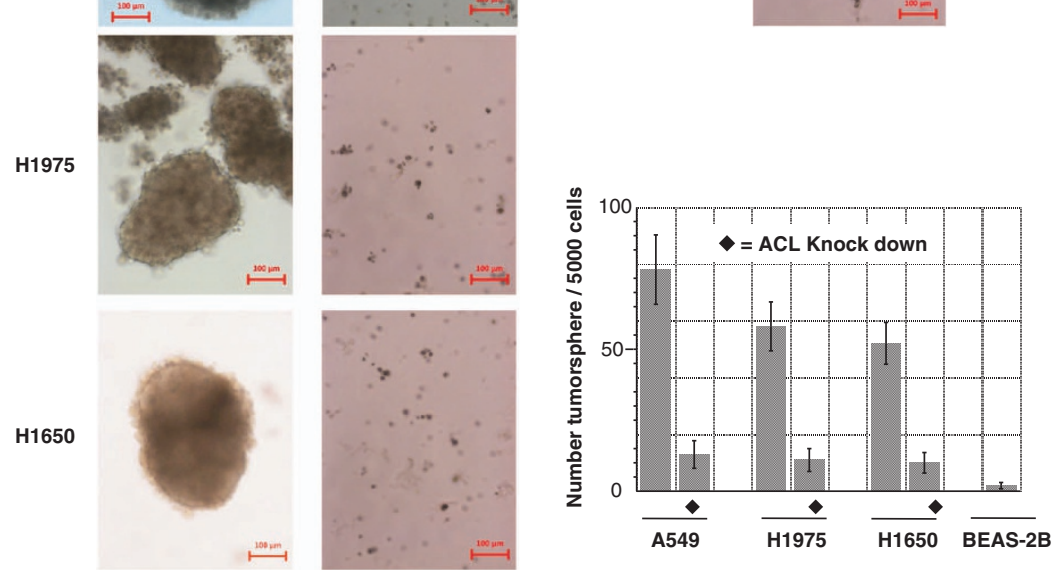

C

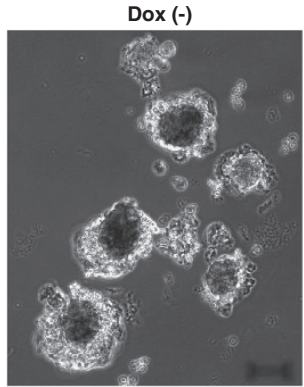

Dox $1.0 \mu \mathrm{g} / \mathrm{ml}$
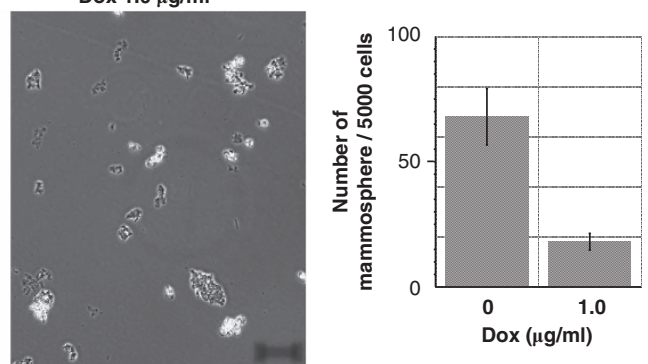

Figure 1 Stem cell populations in non-small-cell lung cancer (NSCLC) cell lines are downregulated by ACL KD. (a) NSCLC cell lines (A549 and H1975) and their ACL KD permanent clones were analyzed by flow cytometry using aldefluor, CD133, CD133/CD34 antibodies or Hoechst 33342 (side population method). (b) Tumorsphere formation assay using NSCLC cell lines (A549, H1975 and H1650) and their ACL KD permanent clones, compared with normal lung epithelial cell line (BEAS-2B). Size bar indicates $100 \mu \mathrm{m}$. (c) Tumorsphere assay using doxycycline inducible ACL KD cells (A549). Size bar indicates $100 \mu \mathrm{m}$ 
a

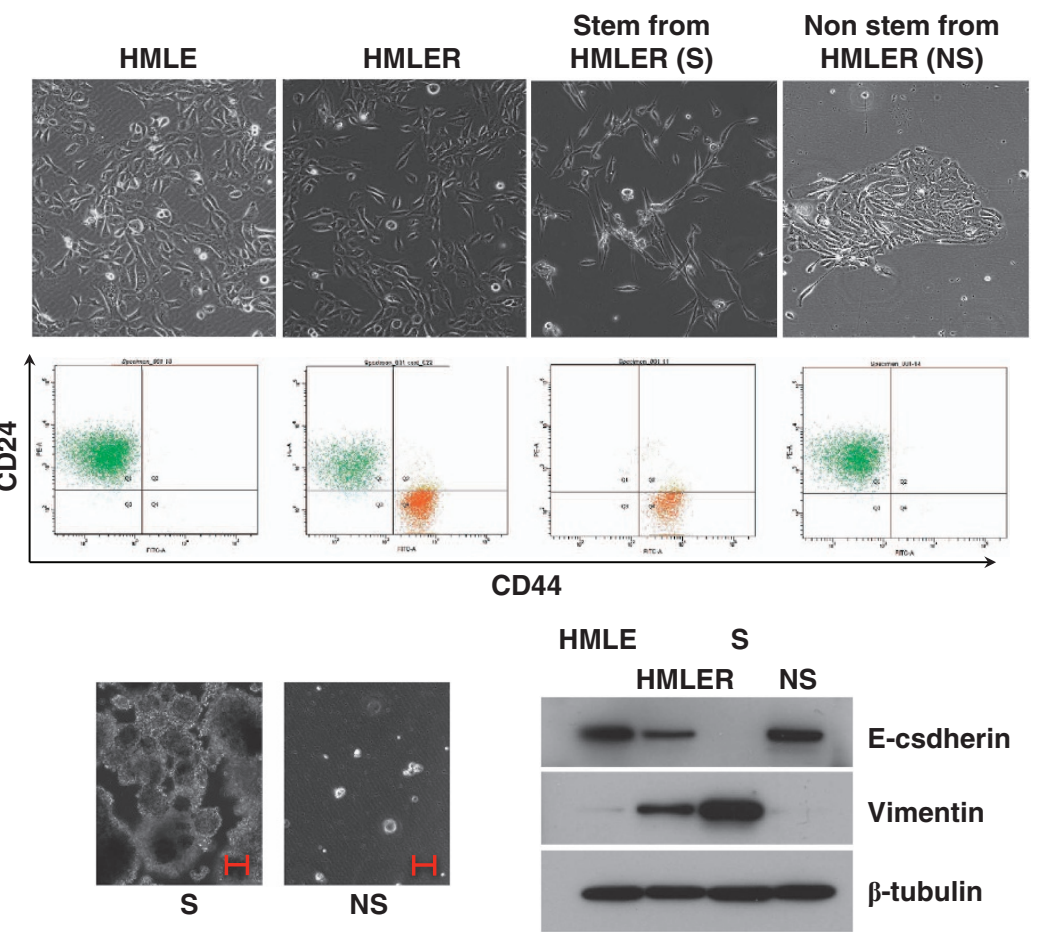

b
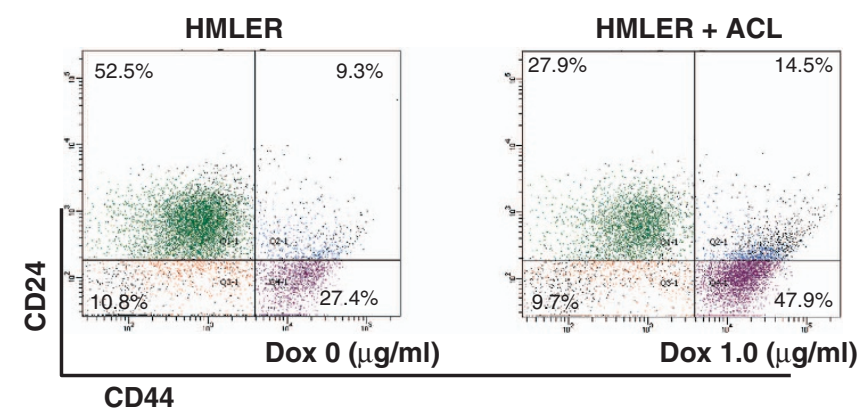

c
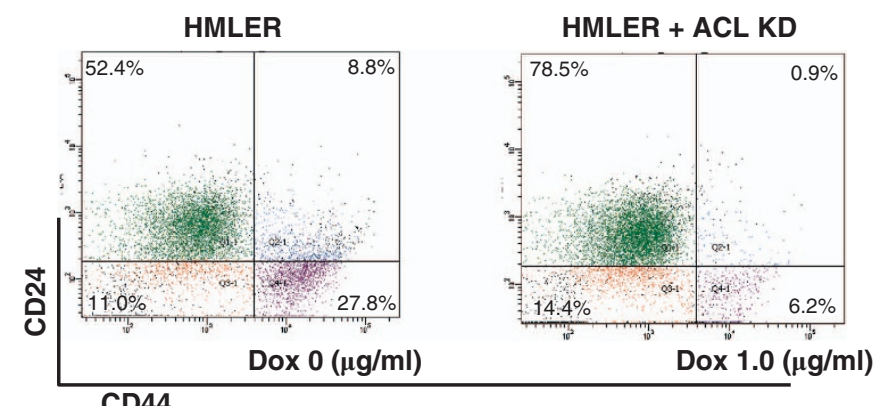

d
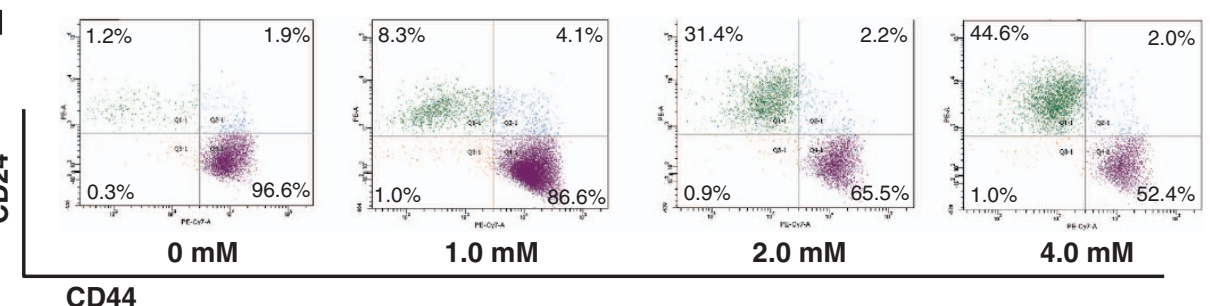

CD44 
inhibitors. There was minimal or no change in transcription factor expression for slug, twist, ZEB1 and ZEB2 by both inhibitors, whereas MEK inhibitor decreased snail protein expression (Figure 3b, Supplementary Figure 4), suggesting that snail might be serving as a target for ACL KD to inhibit Ras-induced stemness in HMLER cells. a

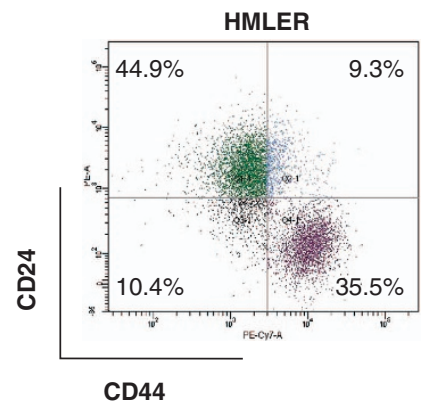

PI3K inhibitor (LY294002)

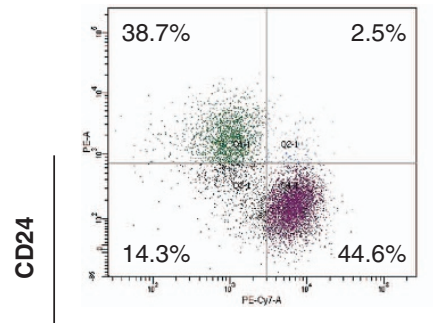

CD44

b

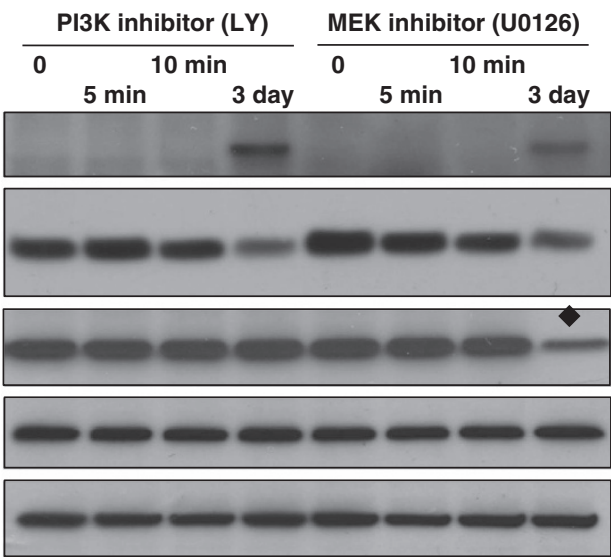

PI3K inhibitor (LY) MEK inhibitor (U0126)

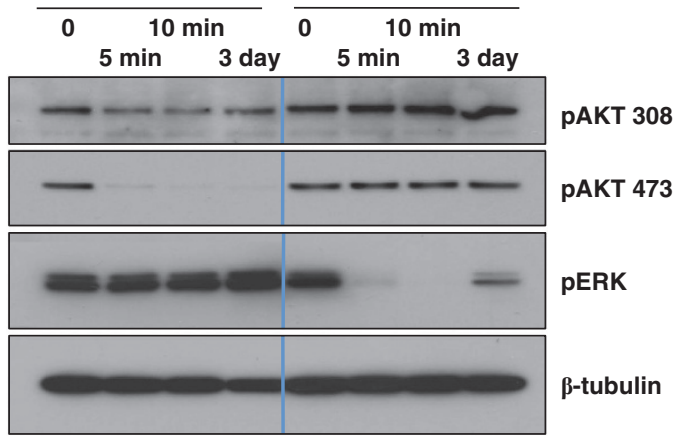

MEK inhibitor (U0126)

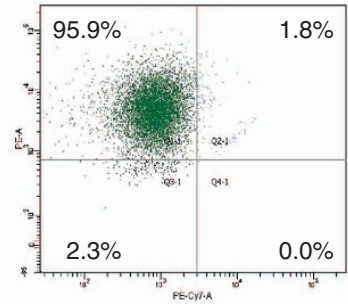

MEK inhibitor (PD035901)

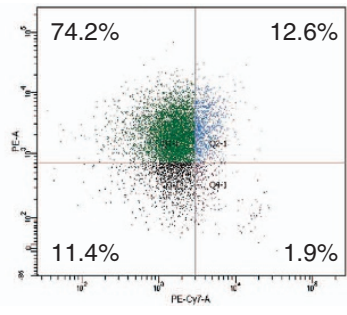

HMLER

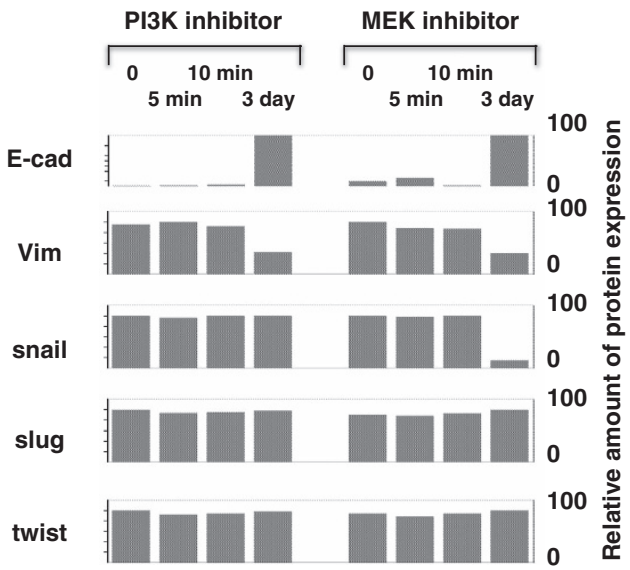

Figure 3 Ras-induced stemness in breast cancer cells (HMLER) is mediated through MAPK. (a) HMLER cells were treated with PI3K inhibitor (LY294002) or MEK inhibitor (U0126 or PD035901) for 3 days and were analyzed by FACS. The activity of each inhibitor was validated by western blot. (b) The effect of each inhibitor on EMT markers and on transcription factors was analyzed by western blot. Both inhibitors reversed EMT but only the MEK inhibitor suppressed snail expression. Snail expression was reduced by MEK inhibitor ( ). The quantitative data are shown on the right side in the graph as a \% of maximum intensity in each category (PI3K inhibitor-treated group and MEK inhibitor-treated group for each protein expression)

Figure 2 Impact of ACL expression on Ras-transformed breast cancer cells. (a) Characteriztion of non-tumorigenic cells (HMLE) and Ras-tarnsformed HMLE (HMLER). FACS analysis of HMLE using CD44 and CD24 antibodies shows a single population, which is, in turn, separated into two populations via Ras-transformation. CD44( + )/ CD24( - ) is a stem cell population (S) and another population (CD44( - )/CD24(+)) is a non-stem population (NS). Each population is isolated separately by FACS sorting and confirmed by mammosphere analysis (size bar indicates $100 \mu \mathrm{m}$.). Morphological analysis and western blot of these $S$ and N populations with original HMLE and HMLER cells demonstrates a clear contrast between mesenchymal (S) and epithelial (NS) cells. (b) Doxycycline inducible ACL overexpression clones are generated in HMLER cells and analyzed by FACS. Induced ACL overexpression upregulated stem cell population. (c) Doxycycline inducible ACL KD clones in HMLER shows reduced stem cell population. (d) S cells (stem cell population sorted out from HMLER cells) were treated with hydroxycitrate (an enzymatic inhibitor of ACL) at the indicated concentration and analyzed by flow cytometry 
Effects of ACL KD on snail-induced stemness. Snail is a downstream regulator of Ras-MAPK ${ }^{26,27}$ signaling and is known to induce stemness in HMLE cells. ${ }^{16}$ To obtain insights into the stemness inhibiting effects of ACL KD, we studied the effects of ACL KD on snail-induced stemness in HMLE cells. Snail overexpressing HMLE cells (E-snail) and ACL KD HMLE-snail cells (E-snail ACL KD) were generated and analyzed by flow cytometry and the mammosphere assay (Figure 4a). E-snail cells manifested an obvious stem cell population in flow cytometry and clear mammosphere formation. ACL KD in these cells (E-snail ACL KD) partially downregulated the stem cell population and partially reduced mammosphere formation. Similarly, treatment with hydroxycitrate also showed partial downregulation of stemness in E-snail cells (Figure 4b).

Association of ACL and snail. Based on the partial effect of ACL KD on snail-induced stemness, we tested for a possible 'association' between snail and ACL. We performed three assays to test snail-ACL 'association': (1) regulation of each's expression by the other, (2) protein-protein interaction and (3) functional cooperation affecting the transcriptional activity of snail.

To address the first issue, ACL overexpressing HMLE cells (E-eACL) were generated (Figure $4 b)$. ACL overexpression in non-tumorigenic HMLE cells (E-eACL) caused no upregulation of snail protein and no upregulation of stemness (data not shown). However, in tumorigenic HMLER cells, ACL overexpression (R-eACL) increased snail expression and increased stemness (Figure 4b). This indicated that ACL itself cannot promote stemness, but in a tumor cell context, ACL overexpression can amplify stemness. On the other hand, snail overexpression in non-tumorigenic HMLE cells (E-snail cells) showed increased ACL expression (Figure 4b) and induced stemness (Figure 4a). This increased stemness was slightly downregulated by ACL KD (E-snail ACL KD; Figure 4a), where snail expression was also reduced (Figure $4 \mathrm{~b}$ ), suggesting that snail could initiate stemness by itself, and ACL expression might regulate snail's function through affecting snail protein expression level. For example, ACL might stabilize snail protein. This idea was further supported by experiments using A549 cells, in which Ras is activated (Figure 4d). Snail protein expression in A549 cells was clearly reduced by $A C L K D$, and snail downregulation by ACL KD was also observed in a non-Ras-dependent context (Supplementary Figure 5). Moreover, retroviral introduction of snail, but not a control vector, induced ACL protein expression (Figure 4d), even when ACL expression was knocked down by shRNA (A549-ACL KD-snail). The ACL expression levels in A549 cells reflect amounts noted in the stem cell population (Figure 4d). The fact that snail overexpression increases $A C L$ expression in ACL-KD cells, may partially explain why ACL-KD was not effective at reversing stemness in snailoverexpressing cells. In the Ras-activated A549 cells, snail expression is clearly regulated by $A C L$ expression level. Reduced snail expression by ACL KD could not be rescued by AKT1 or AKT2 overexpression (Figure 4e), again suggesting that the PI3K/AKT pathway is not of stem cell relevance.

To address whether snail and ACL protein might interact, HMLE, stem cell population (S) from HMLER, E-snail and
E-snail ACL KD cells were processed for immunoprecipitation (IP)-western blotting (WB; Figure 4c). Physical association between endogenous $A C L$ protein and overexpressed snail protein was observed in E-snail cells, where the amounts of both proteins were highest. These data suggest that both proteins may be part of a complex. We speculate that this interaction might be associated with protein stabilization of the snail protein.

To gain more insight into the functional relationship between ACL and snail, we tested the effects of ACL protein expression level on the (inhibitory) transcriptional activity of snail on the human E-cadherin promoter. The E-cadherin promoter activity was monitored in the presence of ACL shRNA using E-snail cells. Transiently transfected ACL shRNA increased E-cadherin promoter activity dose dependently (Figure $4 \mathrm{f}$ left), suggesting that transient ACL KD reduced the transcriptional inhibitory activity of snail. Next, we studied E-cadherin promoter activity using the inducible ACL KD system. Promoter activity was reduced dose dependently by a transiently introduced snail construct, which was rescued by induced ACL KD with doxycycline (Figure $4 \mathrm{f}$ right). In the same system, using different amounts of snail construct and doxycycline, the induced ACL KD, confirmed by WB, clearly counteracted snail's inhibitory activity (Figure $4 \mathrm{~g}$ ). Of note, the expression of snail protein was also reduced in direct proportion to the KD level of ACL expression, indicating that the expression level of $A C L$ regulates snail expression and, in turn, snail transcriptional activity.

Collectively, our data suggest that even though the ACL KD effects were not fully reversing snail-induced stemness, ACL expression impacts snail's function and both proteins might cooperatively function to induce stemness. Snail is, at least partially, one target for ACL KD that has a role in ACL's ability to impact Ras-induced stemness.

Reduced stemness by ACL KD in A549 cells is partially rescued by acetate supplementation. The ACL KD state limits acetyl CoA synthesis from citrate in the cytoplasm. Acetate is the other source of cytoplasmic acetyl CoA, which is synthesized by the ACAS II enzyme. ${ }^{13}$ Cytoplasmic acetyl CoA depletion might be a mechanism by which ACL KD is working, as hydroxycitrate treatment mimicked ACL KD. Thus, we hypothesized that supplementation with acetate might rescue the ACL KD phenotype. This was found to be the case for rescue of $A C L$ function as it relates to histone acetylation, ${ }^{28} \mathrm{PI}$ KK-AKT signaling ${ }^{13}$ and to growth arrest and apoptosis. ${ }^{13,29}$ Here we tested acetate's effect on cancer stemness. As shown in Figure 4e, acetate partially rescued stemness as assessed by tumorsphere formation and FACS analysis using breast CSC markers. Also we assessed the effects of AKT1 and AKT2 overexpression on EMT and stemness. AKT2, but not AKT1, partially rescued EMT (Figure 4e); however, there was no effect on stemness (Figure 4e), consistent with our data using the PI3K inhibitor, showing that there was no effect of inhibiting PI3K-AKT signaling on the maintenance of stemness (Figure $3 a$ ).

Stemness inhibition by ACL KD in other systems. We have already noted that ACL KD can impact stemness in two Ras-driven cell lines (A549 and HMLER) and in two other cell 
a
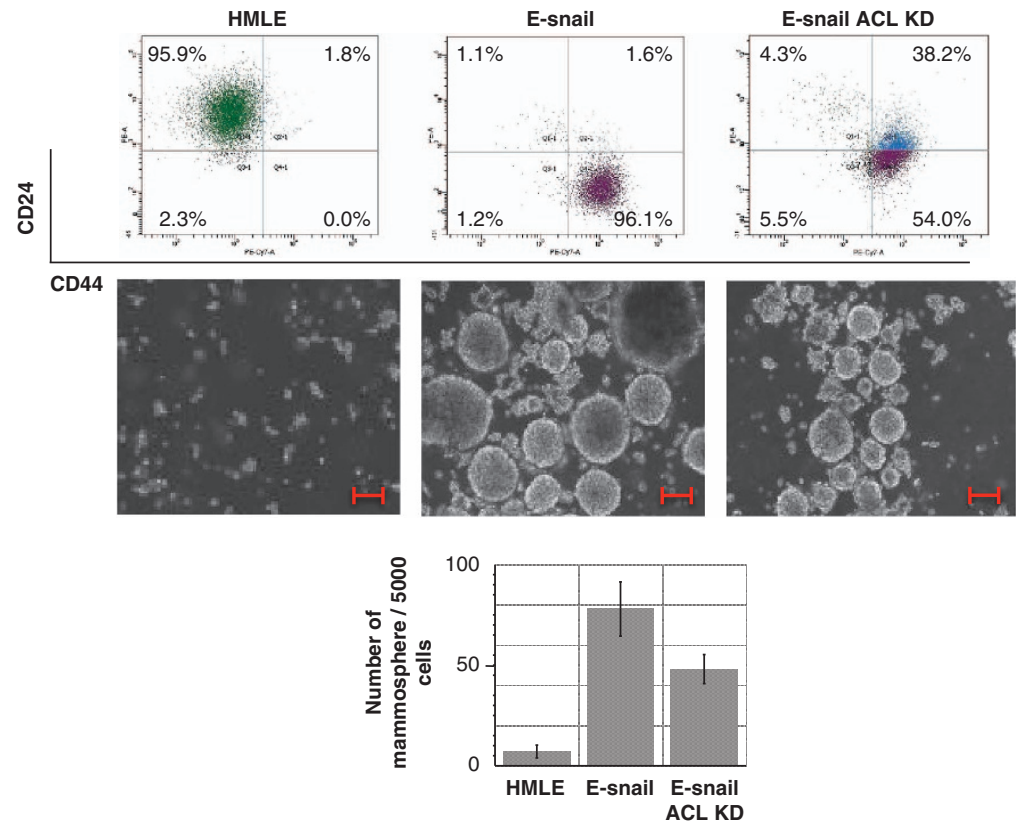

b
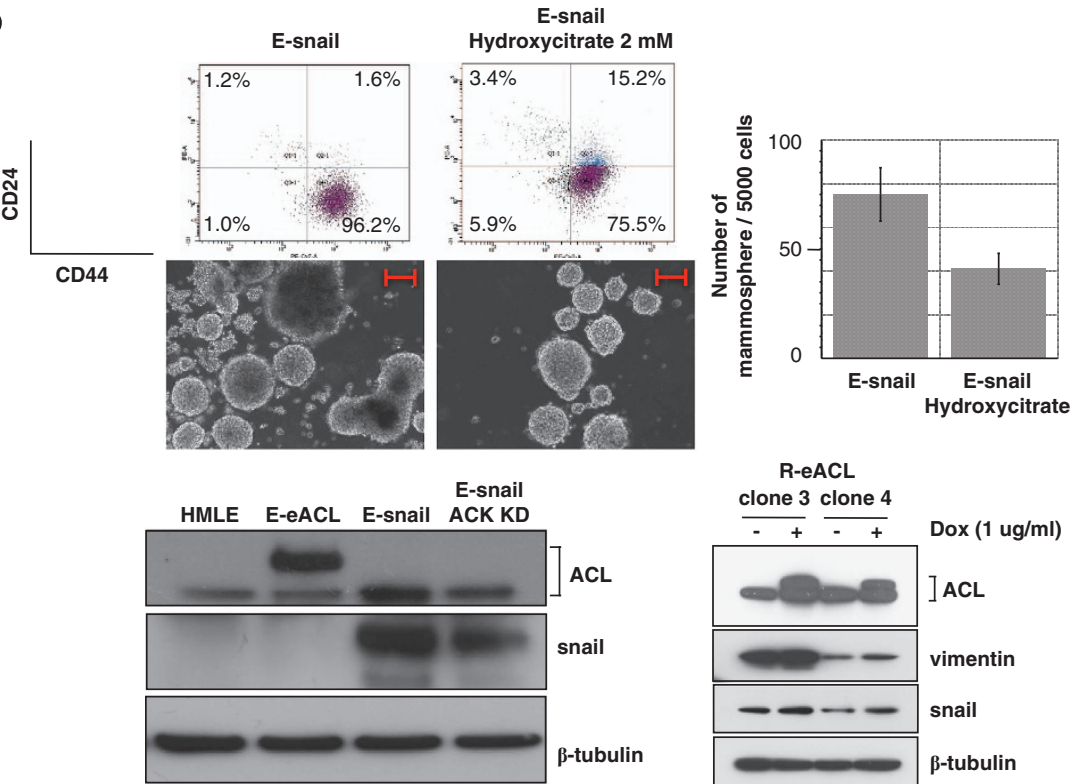

R-eACL

clone 3 clone 4

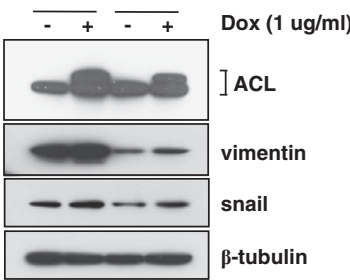

C

IP: IgG

IP: snail

\begin{tabular}{|c|c|c|c|c|c|c|c|}
\hline \multicolumn{4}{|c|}{ IP: IgG } & \multicolumn{4}{|c|}{ IP: snail } \\
\hline HMLE & 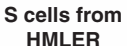 & E-snail & $\begin{array}{l}\text { E-Snail } \\
\text { ACL-KD }\end{array}$ & HMLE & $S$ cells from & E-snail & $\begin{array}{l}\text { E-Snail } \\
\text { ACL-KD }\end{array}$ \\
\hline
\end{tabular}
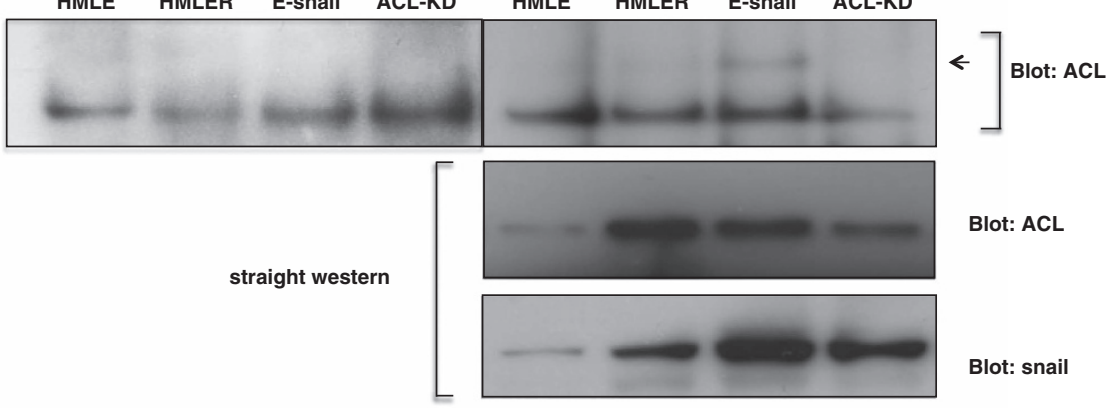

Figure 4 Continued 
d

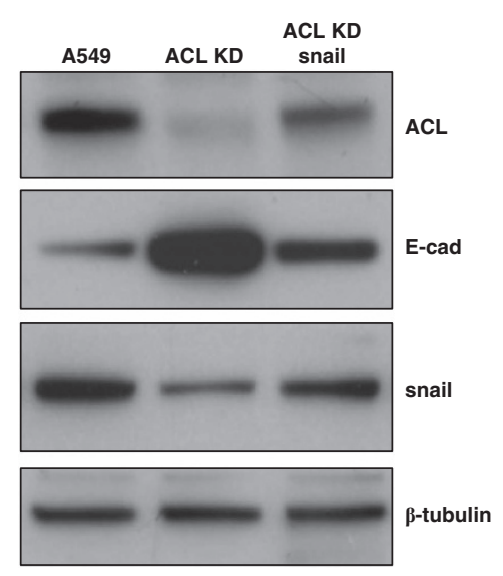

e
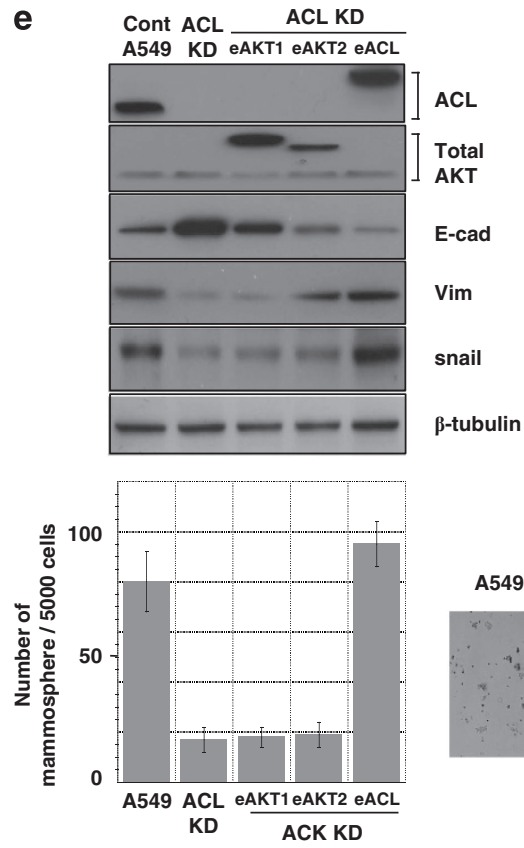
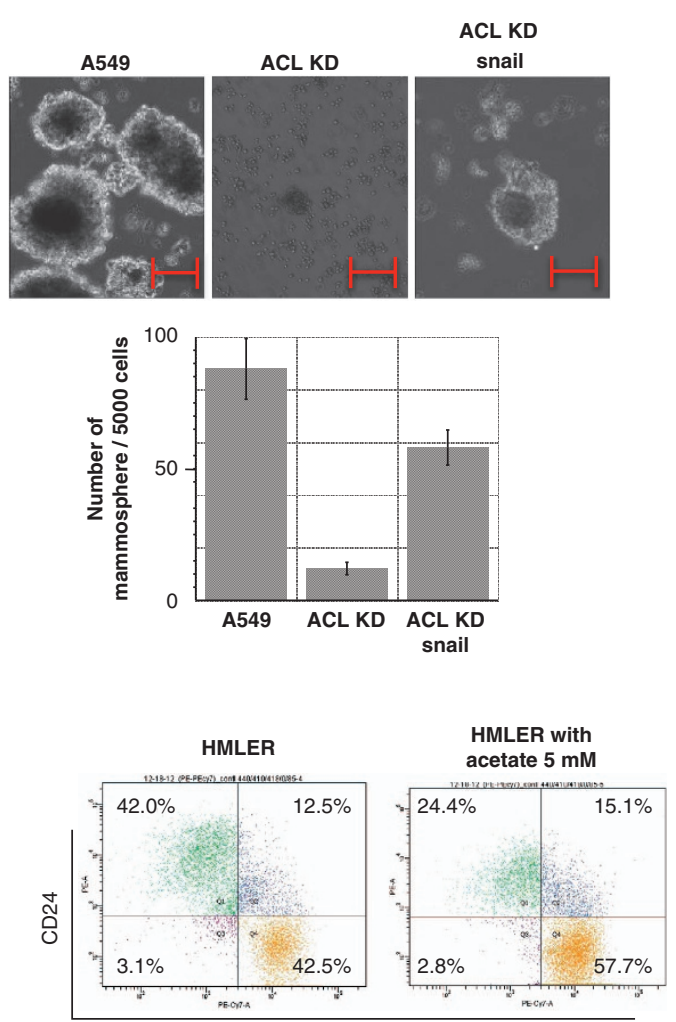

CD44
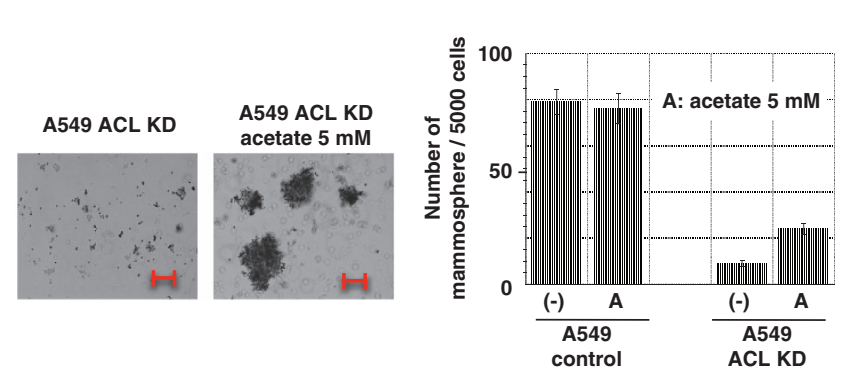

Figure 4 Association between snail and ACL. (a) HMLE, HMLE-snail and HMLE-snail ACL KD cells were processed for FACS and mammosphere analysis (size bar indicates $100 \mu \mathrm{m}$.). (b) HMLE-snail cells treated with or without hydroxycitrate (2 mM) were analyzed by FACS and mammosphere assay (size bar indicates $100 \mu \mathrm{m}$.). (Lower left panel) Inducible ACL overexpressing HMLE cells (E-eACL), Snail stable overexpressed HMLE cells (E-snail) and inducible ACL overexpression plus snail overexpressed HMLE cells (E-snail ACL KD) were compared with HMLE cells for ACL and snail expression levels. ACL overexpression in HMLE could not increase snail expression, but overexpressed snail in HMLE snail cells were downregulated by ACL KD. (Lower right panel) Inducible expression of ACL in HMLER cells (R-eACL) were analyzed by western blot with or without doxycycline induction. Two different clones with different expression levels of vimentin were used. In both clones, induced overexpression of ACL increased snail protein expression. (c) HMLE, stem cell population from HMLER, HMLE-snail and HMLE-snail ACL KD were used for IP-western blotting. Fifty microliters of each cell lysate $(1000 \mu \mathrm{l})$ was processed for western blot of $\mathrm{ACL}$ and snail. The remaining portion of each lysate was immunoprecipitated with control anti-human lgG antibody or snail antibody and blotted with ACL. The arrow indicates the size of ACL protein. Physical association between ACL and snail was observed in HMLE-snail cells, where the amount of snail and ACL expression was highest. (d) A549 control cells, ACL KD cells and ACL KD with overexpressed snail (ACL KD-snail) cells were analyzed by western blot and mammosphere assay (size bar indicates $100 \mu \mathrm{m}$.). Knocked-down ACL expression was rescued by snail overexpression; reduced tumorsphere formation activity by ACL KD was also rescued. (e) (Upper and lower left panel) AKT1, AKT2 or ACL inducible overexpressing clones in ACL KD A549 cells were analyzed by western blot and tumorsphere assay (size bar indicates $100 \mu \mathrm{m}$.). AKT1 or AKT2 overexpression did not rescue downregulated snail expression in ACL KD cells. (Right upper panel) HMLER and HMLER treated with acetate were analyzed by FACS using stem cell markers (upper). (Right lower panel) A549 cells and A549 ACL KD cells were treated with acetate and analyzed by mammosphere assay. ( $\mathbf{f}$ and $\mathbf{g}$ ) Transcriptional analysis of human E-cadherin promoter. E-snail cells were transfected with the ACL KD construct and internal control vector ( $p R L$ TK vector; left panel of e), and A549 inducible ACL KD cells were transfected with snail expression construct and pRL TK vector (right panel of $f$ and $\mathbf{g}$ ). Each cell lysate was analyzed for luciferase activity by luminometer, which was normalized by Renilla luciferase activity (internal control). Expressions levels of snail and ACL (g) at the marked conditions $(\checkmark)$ were confirmed by western blot 
f

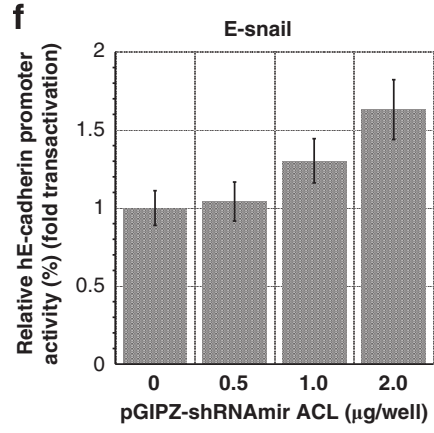

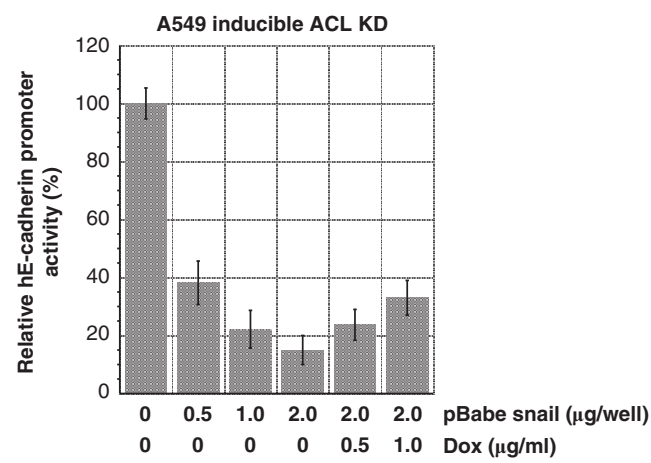

g

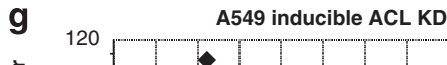

A549 inducible ACL KD

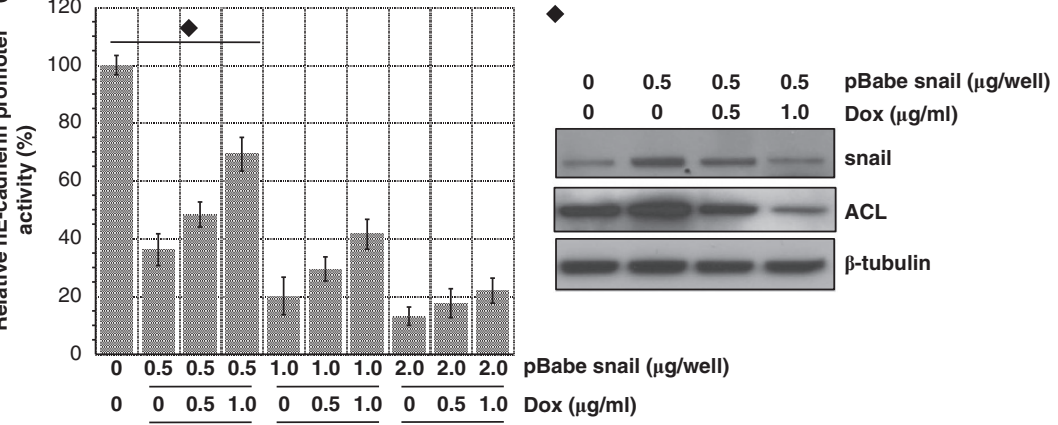

Figure 4 Continued

lines ( $\mathrm{H} 1975$ and $\mathrm{H} 1650)$ that have this pathway activated upstream events (EGFR activation, in this case). Moreover, we have shown in the HMLER system that the Ras-MAPK pathway was critical for maintaining stemness. To test the possibility that $A C L$ KD might impact non-Ras-induced stemness, we obtained cell lines in which stemness is not induced by Ras. ACL KD cells were generated in MCF10A breast epithelial cells stably overexpressing the catalytic domain of PI3K (MCF10A PIK3CA), ${ }^{30}$ MCF10A cells stably overexpressing PTEN shRNA (MCF10A shPTEN), MCF10A cells inducibly overexpressing Src (MCF10A ER-Src) and C8161 invasive melanoma cells (carrying mutated p53). ACL KD cells in these cells showed significant reduction of stemness, as assessed in the tumorsphere assay (Figure 5), suggesting generalized significance of $A C L K D$ for the inhibition of cancer stemness. Importantly, reduction of snail expression was observed in each ACL KD in these cell lines (Figure $5 d$ ), suggesting the snail may, at least partially, be a common 'target' for ACL KD that may mediate its impact on cancer stemness.

Impact of $\mathrm{ACL}$ KD on transcription factors known to induce stemness. A number of transcription factors have been implicated in generating and maintaining stem cell properties. These include c-myc, Klf4, Oct4 and Sox2. We therefore tested whether the expression levels of these factors were influenced by ACL KD. In A549 cells, the greatest effects were seen on the first three of these transcription factors and no impact was noted on Sox2 gene expression (Figure 6). Similar data were noted when ACL was knocked down in E-snail cells (data not shown).

\section{Discussion}

CSCs and metabolism. The connection between metabolism and stemness is a relatively new area of investigation. If we were to uncover metabolic properties that are unique to stem cells and in particular to CSCs, it would provide new targets for cancer therapy. Along these lines, it has recently been reported that the metabolic enzyme glycine decarboxylase is critical for tumor-initiating cells in NSCLC. ${ }^{31}$ There is also an emerging literature on metformin and CSCs, but whether the ability of this biguanide to impact stem cell properties is mediated through activation of AMPK remains to be established. ${ }^{32}$ Here we report that the inhibition of ACL has a major influence on CSCs.

Our study suggests several mechanisms by which ACL inhibition influences stem cells. The fact that administration of hydroxycitrate can mimic the phenotype induced by ACL KD suggests that the enzymatic activity of ACL is critical for stem cell maintenance. In turn, this result suggests that either the accumulation of citrate and/or the depletion of acetate leads to the phenotype. Supporting this hypothesis, we have shown that replenishment of acetate abrogates the phenotype at least partially. We also have unpublished data that administration of citrate can in fact mimic the phenotype of ACL KD (Hanai, Ren, Seth and Sukhatme, unpublished data). It is conceivable that hydroxycitrate acts not only as a competitive inhibitor for ACL but also that it might alter the conformation of $A C L$, thus influencing its interaction with other partners such as snail. Indeed, we have presented evidence that the ACL protein forms a complex with snail, and that it has the ability to alter snail function. 

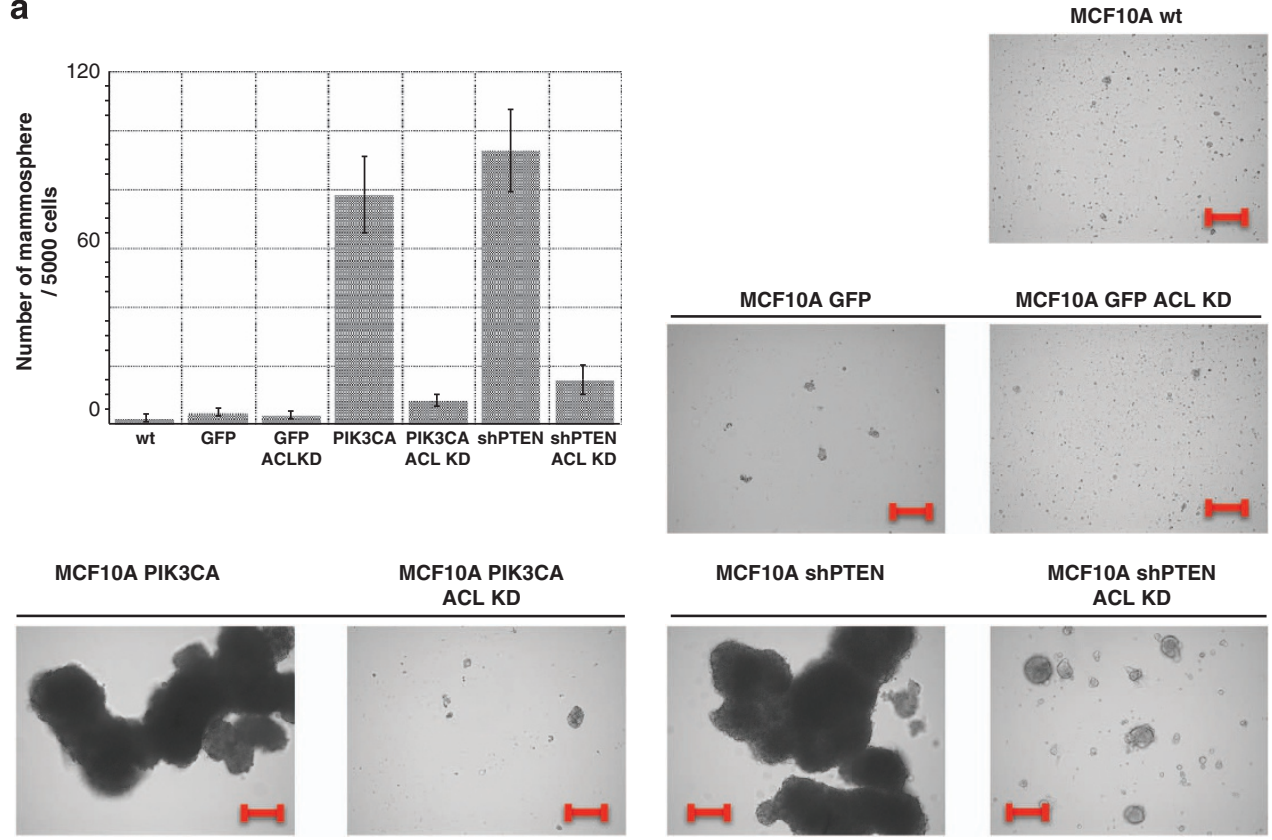

b

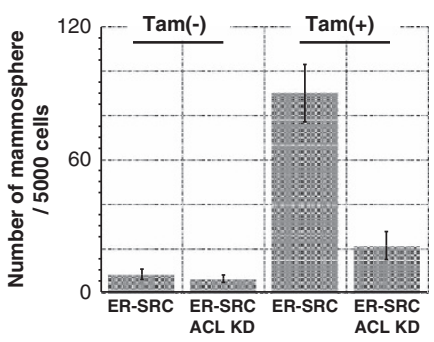

ER-SRC / Tam (-)

ER-SRC ACL KD / Tam (-)
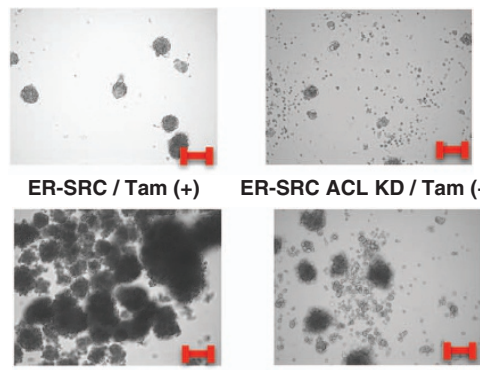

d
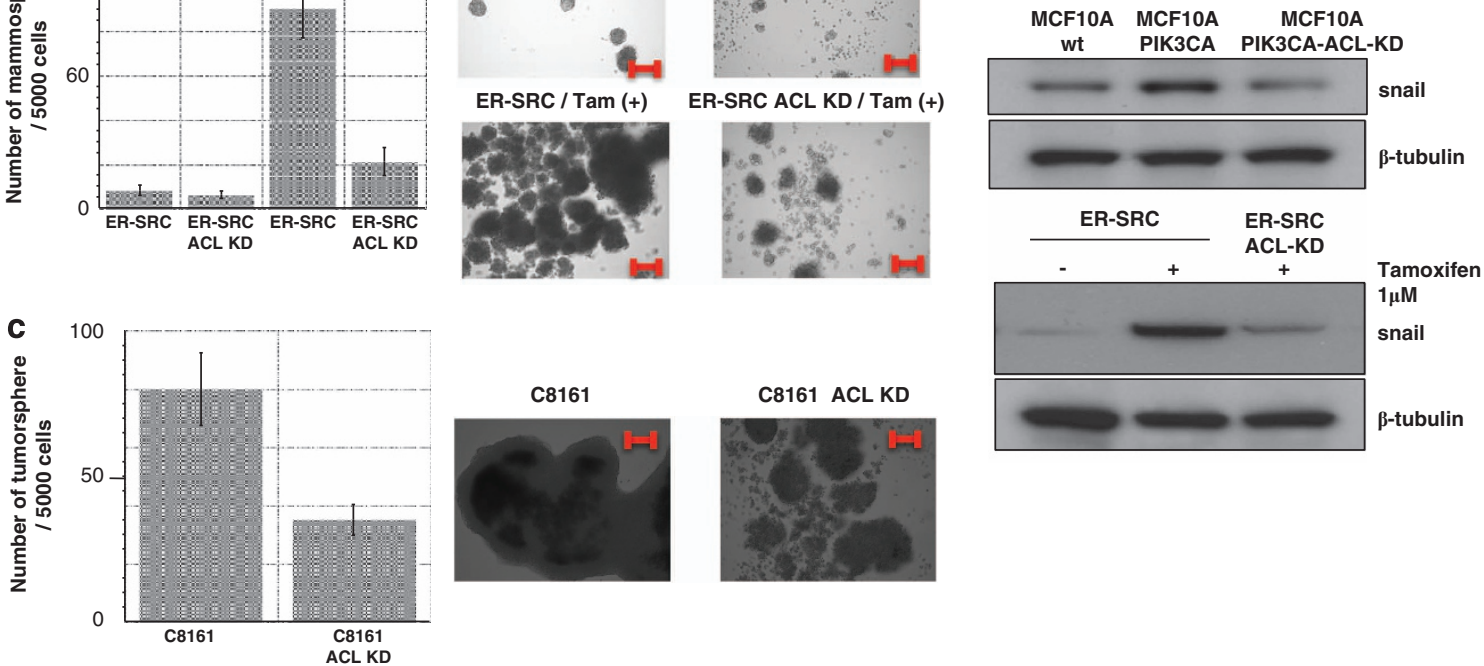

Figure 5 Effects of ACL KD on stemness in other tumorigenic systems. (a) MCF10A cells were stably transfected with PIK3CA (PI3K catalytic unit) or shPTEN (shRNA for PTEN). Cells in these backgrounds with ACL knockdown were analyzed by mammosphere assay and compared with control cells. Size bar indicates $100 \mu \mathrm{m}$. (b) MCF10A cells with ER-Src; ACL knockdown in these cells were analyzed by mammosphere assay. Size bar indicates $100 \mu \mathrm{m}$. (c) C8161 (melanoma) cells and ACL knockdown clones were analyzed by mammosphere assay. Size bar indicates $100 \mu \mathrm{m}$. (d) Diminished snail expression by ACL KD was detected by western blot

Another mechanism by which ACL KD might diminish stemness could be through its effect on glycolysis. Stem cells tend to reside in hypoxic niches and therefore most likely rely on glycolysis followed by fermentation, that is, the Warburg effect for their sustenance. Thompson et al. ${ }^{28}$ noted that glycolysis was inhibited in 3T3-L1 preadipoccytes with ACL $\mathrm{KD}$. It is tempting to speculate that citrate accumulation as a result of $A C L K D$ is inhibiting glycolysis, as citrate is known to affect phosphofructokinase- $1 .{ }^{33}$ Indeed, we have unpublished data that inhibition of the glycolytic pathway can also impact stem cells (Hanai, Seth and Sukhatme, unpublished data).

CSCs and EMT dissociation. In the life of the tumor, stem cells and the EMT process often co-evolve. Extracellular factors, such as TGF- $\beta 1$, and intracellular transcription factors, such as snail, can promote both processes. The net result is a more aggressive tumor phenotype, one that shows a propensity to metastasize, be resistant to 


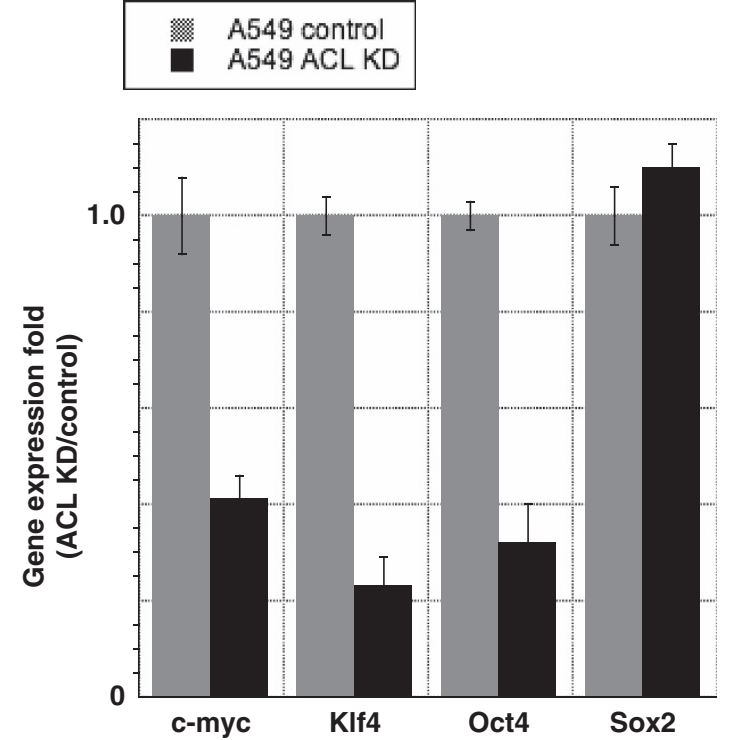

Figure 6 Effects of ACL KD on Yamanaka (pluripotency) factors. Real-time PCR analysis of the indicated factors in A549 control cells and A549 ACL knockdown cells

chemotherapy and to recur. ${ }^{16,34,35}$ Moreover, in some tumor progression models, the acquisition of the characteristics of CSC's is reported to be driven by EMT induction. ${ }^{36}$ We have previously reported that $A C L K D$ in NSCLC induces differentiation through promoting $\mathrm{MET}$, the reverse of EMT. ${ }^{13}$ Therefore, we asked whether ACL KD might downregulate cancer stemness. This was found to be the case, thus uncovering a novel function for ACL. However, to our surprise we found that interruption of the MEK pathway was critical in maintaining the stemness phenotype induced by the Ras oncogene, whereas inhibition of the either MEK pathway or PI3K pathway impacted the EMT process, as indicated by the expression of E-cadherin and vimentin. This dissociation of EMT and stemness is a novel finding.

It is worth noting that a MEK inhibitor has different effects on normal stem cells than what we have found in CSCs. Indeed, MEK inhibition on normal stem cells (induced pluripotent stem cells and embryonic stem cells) maintains self-replication of the pluripotent state and inhibits their commitment to differentiate. ${ }^{30,32,33}$

Translation to the clinic. We do not know how specific the action of ACL inhibition is on CSCs versus normal stem cells. Another limitation of our study is that it is solely in vitro work. Previous in vivo studies by us and others have not evaluated the impact of ACL depletion on CSCs. Moreover, the correct experimental design to maximize the efficacy of such therapies (i.e., reduce tumor burden and prevent recurrence) would be to target both stem and non-stem cell compartments and this was done in the previous studies.

Of note, we have been able to demonstrate that ACL inhibition impacts stemness induced by Ras activation in nonsmall-cell lung cancer and breast cancer lines. Moreover, stemness induced by activation of a number of other oncogenic events such as constitutive activation of EGFR, src, a catalytic subunit of the PI3K, as well as loss of the tumor suppressors $\mathrm{p} 53$ and PTEN are all impacted by ACL inhibition. Moreover, snail expression is also diminished in these systems by ACL inhibition. Given the data describing the interaction of $A C L$ with snail, and the ability of $A C L$ to inhibit snail action, it is conceivable that the underlying mechanism by which ACL inhibits such a broad range of oncogenic and tumor-suppressor activities is through its influence on snail. These results collectively suggest that $\mathrm{ACL}$ inhibition may impact CSCs in a broad range of genetic backgrounds and thus have widespread applicability.

\section{Materials and Methods}

Viral constructs, antibodies and reagents. An empty shRNA vector (pGIPZ) was used as a control and three different ACL shRNA lentiviruses (pGIPZ) were obtained from Open Biosystems (now ThermoFisher Scientific, Cambridge, MA, USA), as previously described. ${ }^{13}$ These shRNAmir target sequences were also cloned from pGIPZ into PTRIPZ (tetracycline-inducible expression vector, Open Biosystems) by a simple restriction digest to generate the pTRIPZ ACL shRNAmir clones, as described previously. ${ }^{13}$ GFP-tagged ACL, AKT1 and AKT2 were generated by the standard PCR method, subcloned into pEGFP-C3 and pLVX-Tight-Puro (Clontech, Mountain View, CA, USA) to generate tetracycline-inducible overexpression lentiviral constructs. Retroviral construct for snail (pBabe-puro-snail) was a gift from Dr. Yoshikawa (Kyoto University). ACL, phospho-AKT 308, phospho-AKT 473, phospho-ERK, AKT1, AKT2, Snail (SN9H2 for WB), Snail (C15D3, for IP) antibodies were purchased from Cell Signaling Technology (Danver, MA, USA). E-cadherin (G-10), vimentin antibodies were from Santa Cruz Biotechnology (Dallas, TX, USA). $\beta$-Tubulin antibody, Hoechst 33342 (Bisbenzimide $\mathrm{H}$ 33342) and (-)-calcium hydroxycitrate tribasic from Sigma-Aldrich (St Louis, MO, USA). Slug and twist antibodies were from Abcam (Cambridge, MA, USA). Glycine dehydrogenase antibody was from Origene (Rockville, MD, USA). CD44-FITC (G44-26), CD44-PE-Cy7, CD24-PE and CD166-FITC antibodies were from BD Biosciences (San Jose, CA, USA), and CD34-FITC, CD133-PE (293C3) antibodies were from Miltenyi Biotec (Auburn, CA, USA), ALDEFLUOR ${ }^{R}$ was obtained from Stem Cell Technologies (Vancouver, BC, Canada). Doxycycline was from Sigma-Aldrich, and MEK inhibitor (U0126), Wortmannin and LY294002 (PI3K inhibitor) were from Cell Signaling Technology.

Cells and cell culture. A549 cells were purchased from the American Type Culture Collection and maintained in Ham's F-12 medium (Mediatech, Dallas, TX, USA) supplemented with $10 \%$ FCS and penicillin/streptomycin (P/S). H1650 and H1975 cells were maintained in RPMI medium (Mediatech) supplemented with $10 \% \mathrm{FCS}$ and P/S. ${ }^{37} 293 \mathrm{FT}$ cells were purchased from Invitrogen (Carlsbad, CA, USA) and maintained in Dulbecco's modified Eagle's medium (DMEM) supplemented with $10 \%$ FCS and P/S supplemented with $1 \mathrm{mM}$ MEM nonessential amino acids, $6 \mathrm{mM}$ L-glutamine, $1 \mathrm{mM}$ sodium pyruvate and $500 \mu \mathrm{g} / \mathrm{ml}$ geneticin. Immortalized human breast epithelial cells (HMLE), which were generated through the introduction of the SV40 large T antigen and hTERT, and tumorigenic human breast epithelial cells, which were generated via retroviral infection of H-RasV12 into HMLE cells (HMLER), were gifts from Dr. Weinberg (Whitehead Institute) and maintained in mammary epithelial growth (MEGM) medium (Biowhittacker, Inc., Maryland, MD, USA); a serum-free medium composed of modified MCDB 170 basal medium with supplements) as described..$^{38}$ All cell lines were grown at $37^{\circ} \mathrm{C}$ in a humidified incubator with $5 \% \mathrm{CO}_{2}$. Cells were grown to $60-70 \%$ confluency, harvested with trypsin and resuspended to the cell density required for each assay.

Generation of modified cell lines via viral infection. $A C L$ stable $K D$ A549 cell line and ACL (doxycycline)-inducible KD A549 or HMLER cell lines were generated as previously described, ${ }^{13}$ using pGIPZ and pTRIPZ constructs. A549, HMLE and HMLER cells overexpressing ACL, AKT1 or AKT2 (doxycycline inducible system) were generated using pLVX-Tet-On and pLVX-Tight-Puro lentiviral constructs (gifts from Drs. Lewis C Cantley and Atsuo Sasaki). Recombinant lentiviral particles were produced by transient transfection of 293FT cells according to a standard protocol. Subconfluent 293FT cells in a 10-cm dish were co-transfected with $3 \mu \mathrm{g}$ of an shRNA plasmid, and $9 \mu \mathrm{g}$ Viral Power packaging mix (an optimized proprietary mix of three plasmids, pLP1, pLP2 and 
pLP/VSVG from Invitrogen) using Lipofectamine 2000 (Invitrogen). After $16 \mathrm{~h}$, the cells were switched to regular growth medium and were allowed to incubate for an additional $48 \mathrm{~h}$. Conditioned cell culture media containing recombinant lentiviral particles was harvested and frozen. Each cell line (A549, HMLE or HMLER) was initially treated with the above cell culture supernatant containing pLVX-Tet-On lentiviral particles for $24 \mathrm{~h}$. These cells were then selected with G418 geneticin (Invitrogen; $500 \mu \mathrm{g} / \mathrm{ml}$ ) to generate stable cell lines with pLVX-Tet-On regulator vector, which were subsequently treated with $\mathrm{pLVX-Tight-Puro} \mathrm{lentiviral} \mathrm{particles}$ containing ACL, AKT1 or AKT2, and selected in puromycin (Invitrogen; $1 \mu \mathrm{g} / \mathrm{ml}$ ) to generate stable cell lines of each inducible overexpressing system. HMLE cells of stably overexpressing snail (HMLE-snail) were generated via retroviral infection. 293FT cells in 1 well of 24 -well-plate (approximately $8 \times 10^{4}$ cells/well) were transfected with $0.2 \mu \mathrm{g}$ of pBabe-puro-snail and $0.2 \mu \mathrm{g}$ of pCL ampho using $1 \mu \mathrm{l}$ of Fugene6 (Roche Applied Science, Branford, CT, USA) and $20 \mu$ of OPTIMEM (Invitrogen) in $1 \mathrm{ml}$ of conditioned medium. Medium was changed from DMEM ( $10 \%$ serum) to DMEM $(10 \%$ serum $)+$ MEGM $(1: 1)$ at $24 \mathrm{~h}$ after transfection and supernatant was collected and filtered using a $0.45-\mu \mathrm{m}$ filter. This $400 \mu \mathrm{l}$ retroviral supernatant was subsequently used for infection with $600 \mu \mathrm{l}$ of MEGM and polybrene (Millipore; $1 \mu \mathrm{l}$ ) for HMLE cells in 1 well of 24-well-plate (approximately $1.2 \times 10^{4}$ cells/well). After $48 \mathrm{~h}$ from infection, HMLE cells were selected with puromycine $(1 \mu \mathrm{g} / \mathrm{ml})$ for $96-120 \mathrm{~h}$. Every generated cell line was validated for each expression by WB analysis. MCF10A cells overexpressing PI3K or shPTEN were gifts from Dr. Tina L Yuan (UCSF Helen Diller Cancer Center) and MCF10A tamoxifen inducible Src overexpressing cells (MCF10A-ER-Src) were provided by Dr. Kevin Struhl (Harvard Medical School).

IP and WB. Cells were washed, scraped and solubilized in a RIPA lysis buffer (Boston BioProducts, Ashland, MA, USA); Tris-HCl $50 \mathrm{mM}, \mathrm{pH}$ 7.4, NaCl $150 \mathrm{mM}$, NP-40 $1 \%$, sodium deoxycholate $0.5 \%$, SDS $0.1 \%$ ) with protease (Roche) and phosphatase (Active Motif, Carlsbad, CA, USA) inhibitor cocktail. After 20 min on ice, the cell lysates were pelleted by centrifugation and the supernatants (cell lysates) were used for IP-WB or directly used for WB. ${ }^{39}$ For IP, cell lysates were pre-cleared by incubation with protein $\mathrm{A} / \mathrm{G}$-agarose beads for $30 \mathrm{~min}$ at $4{ }^{\circ} \mathrm{C}$, then incubated with snail antibody for $2 \mathrm{~h}$, followed by incubation with protein $\mathrm{A} / \mathrm{G}$ Sepharose beads for $30 \mathrm{~min}$ at $4{ }^{\circ} \mathrm{C}$. The beads were washed four times with the RIPA buffer. The immune complexes were then eluted by boiling for $3 \mathrm{~min}$ in the SDS sample buffer containing $10 \mathrm{mM}$ dithiothreitol and subjected to SDS-PAGE. Aliquots of the cell lysates or cell lysates for direct WB were subjected to SDSPAGE without IP. Proteins were electrotransferred to PVDF membranes and immunoblotted with each antibody and detected using Super Signal West Pico Chemiluminescent substrate (Pierce). For re-blotting, the membranes were stripped following the manufacturer's protocol. Each blot shows representative data that was obtained from at least three independent experiments.

Quantitative changes in protein expression were analyzed using densitometry software (ImageJ, NIH), as described previously. ${ }^{13}$

Flow cytometry analysis and FACS. Nonconfluent cultures were trypsinized into single-cell suspension, counted, washed with phosphate-buffered saline (PBS) supplemented with 5\% FBS, and stained with antibodies specific for human cell surface markers as previously described. ${ }^{40,41}$ Briefly, a total of 250000 cells were incubated with antibodies for $25 \mathrm{~min}$ at room temperature. Unbound antibody was washed off and cells were processed for flow cytometry analysis no longer than $1 \mathrm{~h}$ post staining on a Becton Dickinson SORP LSR II, and data were analyzed with CELLQuest Pro software (BD). HMLER cells were FACS-sorted using a Beckman Coulter MoFlo (at BIDMC Flow Cytometry Core) into stem cell population (S: CD44 high and CD24 low) and non-stem cell population (NS: CD44 low and CD24 high). For the detection of CD44, CD44-FITC (G44-26) or CD44-PE-Cy7 was used.

\section{Hoechst 33342 staining and FACS analysis (side population} method). The Hoechst staining was performed as previously described. ${ }^{42}$ Briefly, cells were sieved through a $40-\mu \mathrm{m}$ cell strainer (BD Falcon, San Jose, CA, USA), then the cells were resuspended in conditioned medium (100 000 cells $/ \mathrm{ml})$ and allowed to recover at $37^{\circ} \mathrm{C}$ for $1 \mathrm{~h}$ before treatment with Hoechst. Hoechst 33342 was added at a final concentration of $2.5 \mu \mathrm{g} / \mathrm{ml}$ and incubated at $37^{\circ} \mathrm{C}$ for $1 \mathrm{~h}$. In the control reactions, verapamil (Sigma-Aldrich) was added at a final concentration of $50 \mu \mathrm{g} / \mathrm{ml}$. After incubation, cells were washed once with PBS, and resuspended in PBS supplemented with $5 \%$ FBS. Cells were stained with $1 \mu \mathrm{g} / \mathrm{ml}$ propidium iodide (Sigma-Aldrich) for viability, then analyzed by flow cytometry.
Mammosphere formation assays. Single cells were plated in six-well ultra-low attachment plates (Corning Incorporated, Corning, NY, USA) at a density of 20000 viable cells $/ \mathrm{ml}$. Cells were grown in serum-free MEGM (Biowhittacker, Inc) supplemented with B27 (Invitrogen) and $10 \mathrm{ng} / \mathrm{ml}$ epidermal growth factor (BD Biosciences) as described. ${ }^{43,44}$ Mammospheres were collected by gentle centrifugation ( 800 r.p.m.) after 12 days, and were fractionated by size using $100 \mu \mathrm{m}$ cell strainers (BD Pharmingen) and counted microscopically. Pictures were taken to assess the ratio of spheres to aggregates of cells. To correct for differing growth rates of the cell lines, the average tumorsphere number on day 12 was divided by a proliferation constant for the cell line. To quantify proliferation, cells were seeded on day 0 at 50000 cells/well in a six-well dish in duplicate. Cells were fixed and stained with $0.1 \%$ crystal violet and quantified by extraction in 10\% acetic acid and spectrophotometer reading at optical density $600 \mathrm{~nm}$.

Tumorsphere formation assay. Single-cell suspensions were plated (20 000 cells/well) in six-well ultra-low attachment plates (Corning) in conditioned medium as previously described. ${ }^{41,43}$ Tumorspheres were cultured for 12 days, collected and counted in the same way as for the mammosphere assay.

Transient transfections and transcriptional reporter analysis. Subconfluent $(70-80 \%)$ cells in six-well plates were transfected using FuGENE6 (Roche). Total amount of transfected DNA in each experimental group was adjusted to the same value by adding empty vector DNA. The human E-cadherin promoter $^{45}$ (E-cad-Luc-pGL3, a gift from Dr. Frans Van Roy, VIB-Ghent University) was used as a reporter and pRL TK vector (Renilla luciferase reporter, Promega, Madison, WI, USA) was used as an internal control reporter with combination of pBabe snail or pGIPZ-shRNAmir ACL. After transient transfection of the plasmids, cells were incubated for $20 \mathrm{~h}$ and luciferase activity in the cell lysates was determined by luminometer, using the Dual-Luciferase Reporter Assay System (Promega). The activity was normalized using Renilla luciferase activity under the control of the thymidine kinase promoter. ${ }^{46}$

Statistical analyses. Student's $t$-test was used to evaluate the statistical significance of the results. All values are expressed as Mean \pm S.E.

\section{Conflict of Interest}

The authors declare no conflict of interest.

Acknowledgements. We thank Drs. Barden Chan and Susumu Kobayashi (BIDMC) and members of the Sukhatme laboratory for useful discussions. Also we thank Dr. Tina L. Yuan (UCSF Helen Diller Cancer Center) and Dr. Kevin Struhl (Harvard Medical School) for providing us the modified cell lines of MCF10A and Drs. Lewis C. Cantley (BIDMC), Atsuo Sasaki (University of Cincinnati) and Kiyotsugu Yoshikawa (Kyoto University) for viral constructs. We also thank Dr. Frans Van Roy (VIB-Ghent University) for providing us the human E-cadherin promoter. This work was supported by seed funds from Beth Israel Deaconess Medical Center to VPS.

1. Alison MR, Islam S, Wright NA. Stem cells in cancer: instigators and propagators? J Cell Sci 2010; 123(Pt 14): 2357-2368.

2. Reya T, Morrison SJ, Clarke MF, Weissman IL. Stem cells, cancer, and cancer stem cells. Nature 2001; 414: 105-111.

3. Clarke MF, Dick JE, Dirks PB, Eaves CJ, Jamieson CH, Jones DL et al. Cancer stem cellsperspectives on current status and future directions: AACR Workshop on cancer stem cells. Cancer Res 2006; 66: 9339-9344.

4. Ailles LE, Weissman IL. Cancer stem cells in solid tumors. Curr Opin Biotechnol 2007; 18: 460-466.

5. Chaffer CL, Brueckmann I, Scheel C, Kaestli AJ, Wiggins PA, Rodrigues LO et al. Normal and neoplastic nonstem cells can spontaneously convert to a stem-like state. Proc Natl Acad Sci USA 2011; 108: 7950-7955.

6. Takebe N, Harris PJ, Warren RQ, Ivy SP. Targeting cancer stem cells by inhibiting Wnt, Notch, and Hedgehog pathways. Nat Rev Clin Oncol 2011; 8: 97-106.

7. Dissanayake SK, Wade M, Johnson CE, O'Connell MP, Leotlela PD, French AD et al. The Wnt5A/protein kinase $C$ pathway mediates motility in melanoma cells via the inhibition of metastasis suppressors and initiation of an epithelial to mesenchymal transition. J Biol Chem 2007; 282: 17259-17271.

8. Massague J. TGFbeta in Cancer. Cell 2008; 134: 215-230. 
9. Vincan E, Barker N. The upstream components of the Wnt signalling pathway in the dynamic EMT and MET associated with colorectal cancer progression. Clin Exp Metastasis 2008; 25: 657-663.

10. Yang J, Weinberg RA. Epithelial-mesenchymal transition: at the crossroads of development and tumor metastasis. Dev Cell 2008; 14: 818-829.

11. Thiery JP, Acloque H, Huang RY, Nieto MA. Epithelial-mesenchymal transitions in development and disease. Cell 2009; 139: 871-890.

12. Hung JJ, Yang MH, Hsu HS, Hsu WH, Liu JS, Wu KJ. Prognostic significance of hypoxiainducible factor-1alpha, TWIST1 and Snail expression in resectable non-small cell lung cancer. Thorax 2009; 64:: 1082-1089.

13. Hanai J, Doro N, Sasaki AT, Kobayashi S, Cantley LC, Seth P et al. Inhibition of lung cancer growth: ATP citrate lyase knockdown and statin treatment leads to dual blockade of mitogen-activated protein kinase (MAPK) and phosphatidylinositol-3-kinase (PI3K)/AKT pathways. J Cell Physiol 2012; 227:: 1709-1720.

14. Hatzivassiliou G, Zhao F, Bauer DE, Andreadis C, Shaw AN, Dhanak D et al. ATP citrate lyase inhibition can suppress tumor cell growth. Cancer Cell 2005; 8:: 311-321.

15. Bauer DE, Hatzivassiliou G, Zhao F, Andreadis C, Thompson CB. ATP citrate lyase is an important component of cell growth and transformation. Oncogene 2005; 24:: 6314-6322.

16. Mani SA, Guo W, Liao MJ, Eaton EN, Ayyanan A, Zhou AY et al. The epithelial-mesenchymal transition generates cells with properties of stem cells. Cell 2008; 133: 704-715.

17. Jiang F, Qu Q, Khanna A, Todd NW, Deepak J, Xing L et al. Aldehyde dehydrogenase 1 is a tumor stem cell-associated marker in lung cancer. Mol Cancer Res 2009; 7: 330-338.

18. Moreb JS. Aldehyde dehydrogenase as a marker for stem cells. Curr Stem Cell Res Ther 2008; 3: 237-246.

19. Visvader JE, Lindeman GJ. Cancer stem cells in solid tumours: accumulating evidence and unresolved questions. Nat Rev Cancer 2008; 8: 755-768.

20. Chen YC, Hsu HS, Chen YW, Tsai TH, How CK, Wang CY et al. Oct-4 expression maintained cancer stem-like properties in lung cancer-derived CD133-positive cells. PLoS One 2008; 3: e2637.

21. Eramo A, Lotti F, Sette G, Pilozzi E, Biffoni M, Di Virgilio A et al. Identification and expansion of the tumorigenic lung cancer stem cell population. Cell Death Differ 2008; 15: 504-514.

22. Golebiewska A, Brons NH, Bjerkvig R, Niclou SP. Critical appraisal of the side population assay in stem cell and cancer stem cell research. Cell Stem Cell 2011; 8: 136-147.

23. Hirschmann-Jax C, Foster AE, Wulf GG, Nuchtern JG, Jax TW, Gobel U et al. A distinct 'side population' of cells with high drug efflux capacity in human tumor cells. Proc Natl Acad Sci USA 2004; 101: 14228-14233.

24. Ho MM, Ng AV, Lam S, Hung JY. Side population in human lung cancer cell lines and tumors is enriched with stem-like cancer cells. Cancer Res 2007; 67: 4827-4833.

25. Al-Hajj M, Wicha MS, Benito-Hernandez A, Morrison SJ, Clarke MF. Prospective identification of tumorigenic breast cancer cells. Proc Natl Acad Sci USA 2003; 100 3983-3988.

26. Xiao D, He J. Epithelial mesenchymal transition and lung cancer. J Thorac Dis 2010; 2 : 154-159.

27. Mulholland DJ, Kobayashi N, Ruscetti M, Zhi A, Tran LM, Huang J et al. Pten loss and RAS/MAPK activation cooperate to promote EMT and metastasis initiated from prostate cancer stem/progenitor cells. Cancer Res 2012; 72: 1878-1889.

28. Wellen KE, Hatzivassiliou G, Sachdeva UM, Bui TV, Cross JR, Thompson CB. ATP-citrate lyase links cellular metabolism to histone acetylation. Science 2009; 324: 1076-1080.

29. Zaidi N, Royaux I, Swinnen JV, Smans K. ATP-citrate lyase (ACLY)-knockdown induces growth arrest and apoptosis through different cell- and environment-dependent mechanisms. Mol Cancer Ther 2012; 11: 1925-1935.

30. Ying QL, Wray J, Nichols J, Batlle-Morera L, Doble B, Woodgett J et al. The ground state of embryonic stem cell self-renewal. Nature 2008; 453: 519-523.
31. Zhang WC, Shyh-Chang N, Yang H, Rai A, Umashankar S, Ma S et al. Glycine decarboxylase activity drives non-small cell lung cancer tumor-initiating cells and tumorigenesis. Cell 2012; 148: 259-272.

32. Lin T, Ambasudhan R, Yuan X, Li W, Hilcove S, Abujarour R et al. A chemical platform for improved induction of human iPSCs. Nat Methods 2009; 6: 805-808.

33. Li W, Zhou H, Abujarour R, Zhu S, Young Joo J, Lin T et al. Generation of humaninduced pluripotent stem cells in the absence of exogenous Sox2. Stem Cells 2009; 27: 2992-3000.

34. Gupta PB, Onder TT, Jiang G, Tao K, Kuperwasser C, Weinberg RA et al. Identification of selective inhibitors of cancer stem cells by high-throughput screening. Cell 2009; 138 : 645-659.

35. Wellner U, Schubert J, Burk UC, Schmalhofer O, Zhu F, Sonntag A et al. The EMT-activator ZEB1 promotes tumorigenicity by repressing stemness-inhibiting microRNAs. Nat Cell Biol 2009; 11: 1487-1495.

36. Morel AP, Lievre M, Thomas C, Hinkal G, Ansieau S, Puisieux A. Generation of breast cancer stem cells through epithelial-mesenchymal transition. PLoS One 2008; 3: e2888.

37. Kobayashi S, Boggon TJ, Dayaram T, Janne PA, Kocher O, Meyerson M et al. EGFR mutation and resistance of non-small-cell lung cancer to gefitinib. N Engl J Med 2005; 352: 786-792.

38. Elenbaas B, Spirio L, Koerner F, Fleming MD, Zimonjic DB, Donaher JL et al. Human breast cancer cells generated by oncogenic transformation of primary mammary epithelial cells. Genes Dev 2001; 15: 50-65.

39. Hanai J, Chen LF, Kanno T, Ohtani-Fujita N, Kim WY, Guo WH et al. Interaction and functional cooperation of PEBP2/CBF with Smads. Synergistic induction of the immunoglobulin germline Calpha promoter. J Biol Chem 1999; 274: 31577-31582.

40. Fillmore CM, Gupta PB, Rudnick JA, Caballero S, Keller PJ, Lander ES et al. Estrogen expands breast cancer stem-like cells through paracrine FGF/Tbx3 signaling. Proc Natl Acad Sci USA 107: 21737-21742.

41. Fillmore $\mathrm{CM}$, Kuperwasser $\mathrm{C}$. Human breast cancer cell lines contain stem-like cells that self-renew, give rise to phenotypically diverse progeny and survive chemotherapy. Breast Cancer Res 2008; 10: R25.

42. Storms RW, Goodell MA, Fisher A, Mulligan RC, Smith C. Hoechst dye efflux reveals a novel CD7 ( ) CD34( - ) lymphoid progenitor in human umbilical cord blood. Blood 2000; 96: 2125-2133.

43. Dontu G, Abdallah WM, Foley JM, Jackson KW, Clarke MF, Kawamura MJ et al. In vitro propagation and transcriptional profiling of human mammary stem/progenitor cells. Genes Dev 2003; 17: 1253-1270.

44. Dontu G, Jackson KW, McNicholas E, Kawamura MJ, Abdallah WM, Wicha MS. Role of Notch signaling in cell-fate determination of human mammary stem/progenitor cells. Breast Cancer Res 2004; 6: R605-R615.

45. Comijn J, Berx G, Vermassen P, Verschueren K, van Grunsven L, Bruyneel E et al. The two-handed $\mathrm{E}$ box binding zinc finger protein SIP1 downregulates $\mathrm{E}$-cadherin and induces invasion. Mol Cell 2001; 7: 1267-1278.

46. Hanai J, Gloy J, Karumanchi SA, Kale S, Tang J, Hu G et al. Endostatin is a potential inhibitor of Wnt signaling. J Cell Biol 2002; 158: 529-539.

(i) (s) $\ominus$ Cell Death and Disease is an open-access journal published by Nature Publishing Group. This work is licensed under a Creative Commons Attribution-NonCommercialNoDerivs 3.0 Unported License. To view a copy of this license, visit http://creativecommons.org/licenses/by-nc-nd/3.0/ 